\title{
The Hypercentral Constituent Quark Model
}

\author{
M. M. Giannini, E. Santopinto, A. Vassallo \\ Dipartimento di Fisica dell'Universita' di Genova and I.N.F.N. Sezione di Genova
}

November 1, 2018

\begin{abstract}
The hypercentral constituent quark model contains a spin independent three-quark interaction inspired by lattice QCD calculations which reproduces the average energy of $S U(6)$ multiplets [1]. The splittings are obtained with a residual generalized $S U(6)$-breaking interaction including an isospin dependent term [2]. The long standing problem of the Roper is absent and all the 3- and 4-star states are well reproduced. The model has been used in a systematic way for transverse and longitudinal electromagnetic transition form factor of the 3- and 4- star and also for the missing resonances. The prediction of the electromagnetic helicity amplitudes agrees quite well with the data except for low $Q^{2}$, showing that it can supply a realistic set of quark wave functions. In particular we report the calculated helicity amplitude $A_{1 / 2}$ for the $S 11(1535)$, which is in agreement with the TJNAF data [3].
\end{abstract}

\section{Introduction}

The baryon spectrum is usually described quite well by different Constituent Quark Models [1, 4, 5], which have in common the three constituent quark spatial degrees of freedom and the underlying SU(6) spin-flavour structure. However they differ for the chosen potential, that is for the wave functions, and in particular also for the way in which the $S U(6)$ symmetry is broken. By comparing various models one can see that each of them has different predictions concerning the position and number of the missing resonances under $2 \mathrm{GeV}$, giving the possibility of discriminate among them, but due to the difficulties of the analysis of the experimental data this is not always possible. In this respect the study of hadron spectroscopy is not sufficient to distinguish among the various forms of quark dynamics and to this end one has to consider other observables more sensitive to the wave functions and 
to the way in which $\mathrm{SU}(6)$ is broken such as the e.m. transition form factors, the so called helicity amplitudes.

\section{The Model}

The hypercentral Constituent Quark Model (hCQM) consists of a hypercentral quark interaction containing a linear plus coulomb-like term, as suggested by lattice QCD calculations [7]

$$
V(x)=-\frac{\tau}{x}+\alpha x, \text { with } x=\sqrt{\rho^{2}+\lambda^{2}},
$$

where $x$ is the hyperradius defined in terms of the standard Jacobi coordinates $\rho$ and $\boldsymbol{\lambda}$. We can think of this potential both as a two body potential in the hypercentral approximation (which, as shown by Fabre de la Ripelle [8], is a good approximation for the lower states) or as a true three body potential. A hyperfine term of the standard form [4] is added and treated as a perturbation. The parameters $\alpha, \tau$ and the strength of the hyperfine interaction are fitted to the spectrum $\left(\alpha=1.61 \mathrm{fm}^{-2}, \tau=4.59\right.$ and the strength of the hyperfine interaction is determined by the $\Delta$ - Nucleon mass difference). Having fixed the values of these parameters, the resulting wave functions have been used for the calculation of the photocouplings [9], the transition form factors for the negative parity resonances [10], the elastic form factors [11] and the ratio between the electric and magnetic form factors of the proton [12] and now also for the longitudinal and transition form factors for all the 3- and 4-stars and the missing resonances [13].

\section{The electromagnetic transition form fac- tors}

The helicity amplitudes for the electroexcitation of baryon resonances, $A_{1 / 2}$, $A_{3 / 2}$ and $S_{1 / 2}$ are calculated as the transition matrix elements of the transverse and longitudinal electromagnetic interaction between the nucleon and the resonance states given by this model. A non relativistic current for point quarks is used. In particular, in Fig.1, one can see the predictions (full line) for the $A_{1 / 2}^{p}$ for the $S 11(1535)$ [10] which are in agreement with the new TJNAF data [3]. The results for the helicity amplitudes for all the negative parity resonances are reported in Ref.[10]. The prediction of the electromagnetic helicity amplitudes agrees quite well with the data showing that the hCQM can supply a realistic set of quark wave functions. In general the $Q^{2}$ behaviour is reproduced, except for discrepancies at small $Q^{2}$, specially in the $A_{3 / 2}^{p}$ amplitude of the transition to the $D_{13}(1520)$ state. These discrepancies could be ascribed to the non-relativistic character of the model, 


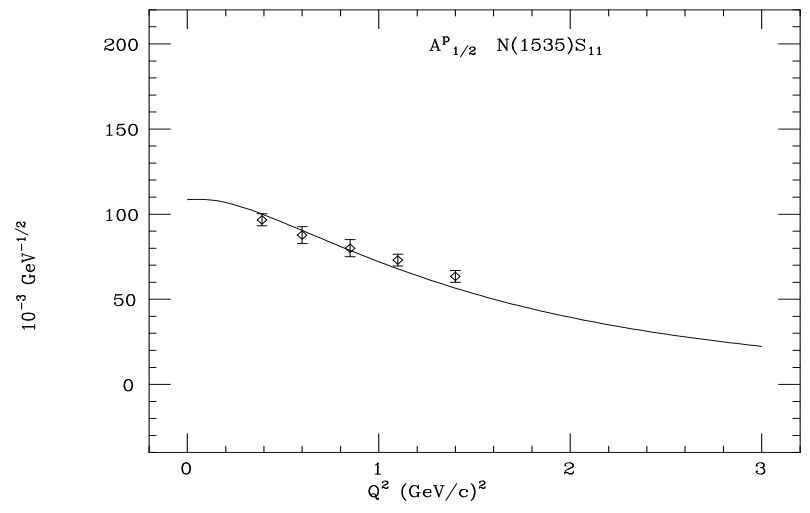

Figure 1: Comparison between the new TJNAF data for the helicity amplitudes $A_{1 / 2}^{p}$ for the $S_{11}(1535)$ [3] and the calculations with the hCQM[9].

and to the lack of explicit quark-antiquark configurations which may be important at low $Q^{2}$. The longitudinal and transverse transition form factors for all the 3-and 4-stars and the missing resonances have been calculated [13]. The computer code is at disposal under request and it can be used also with other models. In this way it can be a useful tool for the forthcoming analysis of the experimental data.

The relativistic corrections at the level of boosting the nucleon and the resonances states to the EVF or the Breit frame are important but not sufficient [15]. These boost effects are indeed important for the elastic e.m. form factors, giving origin to the decreasing behaviour of the ratio $\mathrm{R}$ of the electric and magnetic proton form factors, as shown by the recent TJNAF data [16].

\section{$4 S U(6)$-breaking residual interaction.}

There are different motivations for the introduction of a residual flavour dependent term in the three-quark interaction. The well known GuerseyRadicati mass formula [17] contains a flavour dependent term, which is essential for the description of the strange spectrum. In the chiral Constituent Quark Model [6], the non confining part of the potential is provided by the interaction with the Goldstone bosons, giving rise to a spinand isospin-dependent interaction. More generally, one can expect that the quark-antiquark pair production can lead to an effective residual quark interaction containing an isospin (or flavour) dependent term and with these motivations in mind, we have introduced [2] isospin dependent terms in the 
hCQM hamiltonian. The complete interaction used is given by

$$
H_{\text {int }}=V(x)+H_{\mathrm{S}}+H_{\mathrm{I}}+H_{\mathrm{SI}},
$$

were $\mathrm{V}(\mathrm{x})$ is the linear plus hypercoulomb $\mathrm{SU}(6)$-invariant potential, already described in Eq.(1), while $H_{\mathrm{S}}+H_{\mathrm{I}}+H_{\mathrm{SI}}$, is a residual $\mathrm{SU}(6)$-breaking interaction that can be treated as a perturbative term leading to an improved description of the spectrum.

\section{Conclusions}

We have presented various results predicted by the hypercentral Constituent Model compared with the experimental data. We have shown that the hCQM can supply a realistic set of quark wave functions.

\section{References}

[1] M. Ferraris, M.M. Giannini, M. Pizzo, E. Santopinto and L. Tiator, Phys. Lett.B364, 231 (1995).

[2] M.M. Giannini,E. Santopinto,A. Vassallo, Eur.Phys.J. A12, 447 (2001); Nucl.Phys.A699,308 (2002).

[3] R.A. Thompson et al., Phys. Rev. Lett. 86, 1702 (2001).

[4] N. Isgur and G. Karl, Phys. Rev. D18, 4187 (1978); D19, 2653 (1979); D20, 1191 (1979); S. Godfrey and N. Isgur, Phys. Rev. D32, 189 (1985);

[5] S. Capstick and N. Isgur, Phys. Rev. D 34,2809 (1986).

[6] L. Ya. Glozman, et al., Phys. Rev. C57, 3406 (1998).

[7] Gunnar S. Bali, Phys. Rep. 343, 1 (2001).

[8] M. Fabre de la Ripelle and J. Navarro, Ann. Phys.(N.Y.)123, 185 (1979).

[9] M. Aiello, M. Ferraris, M.M. Giannini, M. Pizzo and E. Santopinto, Phys. Lett. B387, 215 (1996).

[10] M. Aiello, M. M. Giannini, E. Santopinto, J. Phys. G: Nucl. Part. Phys. 24, 753 (1998)

[11] M. De Sanctis, E. Santopinto, M.M. Giannini, Eur. Phys. J. A1, 187 (1998).

[12] M. De Sanctis, M.M. Giannini, L. Repetto, E. Santopinto, Phys. Rev. C62,025208 (2000). 
[13] to be published.

[14] E. Santopinto, F. Iachello and M.M. Giannini, Nucl. Phys. A623, 100c (1997); Eur. Phys. J. A1,307 (1998).

[15] M. De Sanctis, E. Santopinto, M.M. Giannini, Eur. Phys. J. A2, 403 (1998).

[16] M.K. Jones et al., Phys. Rev. Lett. B84,1398 (2000).

[17] F. Guersey and L.A. Radicati, Phys. Rev. Lett. 13, 173 (1964);

[18] Particle Data Group, Eur. Phys. J. C15, 1 (2000). 